\title{
GluR6-FasL-Trx2 mediates denitrosylation and activation of procaspase-3 in cerebral ischemia/reperfusion in rats
}

\author{
N Sun ${ }^{1}$, J-R Hao ${ }^{1}$ X-Y Li ${ }^{2}$, X-H Yin ${ }^{2}$, Y-Y Zong ${ }^{2}$, G-Y Zhang ${ }^{2, \text {, }}$ and C Gao*,1
}

Global cerebral ischemia/reperfusion (I/R) facilitates the activation of procaspase-3 and promotes apoptosis in hippocampus. But the mechanisms have remained uncharacterized. Protein S-nitrosylation and denitrosylation is an important reversible posttranslational modification, which is a common mechanism in signal transduction and affects numerous physiological and pathophysiological events. However, it is not known whether S-nitrosylation/denitrosylation modification of procaspase-3 serves as a component of apoptosis and cell death induced by cerebral I/R. Here we show that procaspase-3 is significantly denitrosylated and activated after I/R in rat hippocampus. NS102, a glutamate receptor 6 (GluR6) antagonist, can inhibit the denitrosylation of procaspase-3 and diminish the increased Fas ligand (FasL) and thioredoxin (Trx)-2 expression induced by cerebral I/R. Moreover, downregulation of FasL expression by antisense oligodeoxynucleotides inhibits the denitrosylation and activation of procaspase-3. Auranofin, a TrxR inhibitor or TrxR2 antisense oligodeoxynucleotide, has similar effects. In primary hippocampal cultures, Lentiviral-mediated knockdown of FasL and TrxR2 before the oxygen and glucose deprivation/reoxygenation further verifies that FasL and TrxR2 are involved in the denitrosylation of procaspase-3. In situ TUNEL staining and cresyl violet staining validate that inhibiting denitrosylation of procaspase-3 may exert neuroprotective effect on apoptosis and cell death induced by cerebral I/R in hippocampal CA1 pyramidal neurons. This is the first evidence that cerebral I/R mediates procaspase-3 denitrosylation and activation through GluR6-FasL-Trx2 pathway, which leads to neuronal apoptosis and cell death.

Cell Death and Disease (2013) 4, e771; doi:10.1038/cddis.2013.299; published online 15 August 2013

Subject Category: Neuroscience

S-Nitrosylation and denitrosylation, a redox-based modification, have a pivotal role in signal transduction by the mechanisms of covalent attachment that control the addition or the removal of nitric oxide (NO) from a cysteine thiol. ${ }^{1} \mathrm{NO}$ is generated from L-arginine in a reaction catalyzed by $\mathrm{NO}$ synthases (NOSs) including neuronal NOS, endothelial NOS, and inducible NOS. ${ }^{2,3}$ Protein S-nitrosylation is a process, in which a NO group is added to the protein cysteine thiol without enzyme-catalyzed reaction. ${ }^{4,5}$ In contrast to S-nitrosylation, two specific enzymatic systems of protein denitrosylation have recently been described. One is the S-nitrosogulutathione reductase (GSNOR) system including $\mathrm{GSH}$ and GSNOR, the other is the thioredoxin (Trx) system comprising Trx proteins, Trx reductase (TrxR) proteins, and nicotinamide adenine dinucleotide phosphate (NADPH). Theoretically, the denitrosylation of target proteins is mediated either by catalyzing the conversion of reduced $\mathrm{Trx}-(\mathrm{SH})_{2}$ to oxidized Trx-S $\mathrm{S}_{2}$ or by GSH. Trx-S $\mathrm{S}_{2}$ is reduced by NADPH, catalyzed by TrxR. ${ }^{6,7}$ TrxR inhibitor auranofin blocks reduced oxidized thioredoxin from S-nitrosylation protein. ${ }^{8}$
Caspase-3 has an important role as a downstream member of the protease cascades, where various cell death pathways converge into the same effecter pathway. ${ }^{9}$ Procaspase- 3 , the caspase-3 proenzyme, has been found S-nitrosylated at Cys163 in resting human cell lines and denitrosylated upon Fas stimulation. ${ }^{10}$ This is the only site that has been demonstrated to be S-nitrosylated in procaspase-3 so far. Procaspase- 3 is cleaved into its active subunits (P17 and P12) after denitrosylation-promoting cell apoptosis. ${ }^{11}$ S-Nitrosylated/denitrosylated procaspse-3 holds important function in cellular apoptosis signaling and transduction pathway. ${ }^{12-15}$

Our previous researches have showed that global cerebral ischemia/reperfusion (I/R) can promote the combination of glutamate receptor 6 (GluR6) and mixed lineage kinase 3 (MLK3) with postsynaptic density (PSD)-95 to facilitate the activation of c-Jun N-terminal kinase (JNK) in rat hippocampus. ${ }^{16-19}$ Activated JNK may translocate into the nucleus leading to cell apoptosis by phosphorylating C-Jun and enhancing the expression of Fas ligand (FasL). ${ }^{17,20}$ But the downstream of FasL-mediated activation of caspase-3 as well

\footnotetext{
${ }^{1}$ Jiangsu Key Laboratory of Anesthesiology, Xuzhou Medical College, Jiangsu 221004, China and ${ }^{2}$ Research Center for Biochemistry and Molecular Biology, Jiangsu Key Laboratory of Brain Disease Bioinformation, Xuzhou Medical College, 209 Tongshan Road, Jiangsu 221004, China

*Corresponding author: C Gao, Jiangsu Key Laboratory of Anesthesiology, Xuzhou Medical College, 209 Tongshan Road, Jiangsu 221004 , China. Tel: + 86-516-83262693; Fax: + 86516 83262686, E-mail: gaocan@xzmc.edu.cn

"Deceased.

Keywords: brain ischemic; FasL; Trx2; Procaspase-3; denitrosylation; neuroprotection.

Abbreviations: I/R, ischemia/reperfusion; GluR6, glutamate receptor 6; FasL, Fas ligand; NOSs, NO synthases; GSNOR, S-nitrosogulutathione reductase; Trx, thioredoxin; TrxR, Trx reductase; NADPH, nicotinamide adenine dinucleotide phosphate; MLK3, mixed lineage kinase 3; PSD, postsynaptic density; JNK, C-Jun $\mathrm{N}$-terminal kinase; shRNAs, short hairprin RNAs; FADD, Fas-associating protein with death domain; OGD/R, oxygen and glucose deprivation/reoxygenation; NO, nitric oxide; DMSO, dimethylsulfoxide; MMTS, methyl methylthiomethyl sulfoxide; SDS-PAGE, SDS-polyacrylamide gel electrophoresis

Received 22.4.13; revised 10.7.13; accepted 15.7.13; Edited by A Verkhratsky
} 
as the apoptosis have not been studied. In the present study, we show that cerebral I/R increase expressions of FasL and Trx2 through GluR6, which in turn denitrosylates procaspase-3. Actived caspase-3 then induces apoptosis in hippocampus. GluR6-FasL-Trx2 signal pathway induces procaspase-3 denitrosylation and activation leading to hippocampal neuronal apoptosis upon I/R. Inhibiting denitrosylation of procaspase-3 may exert neuroprotective effect on apoptosis and cell death induced by cerebral I/R.

\section{Results}

The denitrosylation and activation of procaspase-3 during cerebral I/R. To investigate whether procaspase-3 is activated through denitrosylation in hippocampus upon cerebral I/R, we examined the time course of procaspase-3 $S$-nitrosylation (Figure 1a) and activation (Figure 1b) in rats. Procaspase-3 S-nitrosylation was significantly reduced after $3 \mathrm{~h} \mathrm{I} / \mathrm{R}$ in CA1 region, whereas cleaved caspase-3, the activated form of procaspase-3, was considerably increased and reached peak levels at $6 \mathrm{~h} \mathrm{I} / \mathrm{R}$. The results indicate that part of procaspase- 3 is activated through denitrosylation upon I/R in hippocampal CA1 area.

\section{Cerebral I/R induce procaspase-3 denitrosylation and activation via GluR6 subunit-containing Kainate receptors. Our previous researches have demonstrated that GluR6-KA receptor has an important role in brain ischemia. ${ }^{17,20}$ To explore the main mechanism for procas- pase-3 denitrosylation and activation, rats were pretreated with NS102, an antagonist of GluR6 subunit. The present results showed that NS102 almost prevented the denitrosy- lation (Figure 2a) and activation (Figure $2 \mathrm{~b}$ ) of procaspase-3, which concluded that KA receptor GluR6 subunit was involved in this process of procaspase-3 activation. Further- more, NS102 could inhibit the increased expression of FasL (Figure 2c) and Trx-2 (Figure 2d) induced by $6 \mathrm{~h} \mathrm{I/R}$ in CA1 region. These results suggest that the Fas pathway and Trx-2 system may have a role in GluR6-KA receptor-coupled procaspase-3 denitrosylation.}

\begin{abstract}
JNK mediates denitrosylation and activation of procaspase-3during I/R. Our previous studies have clarified that I/R can facilitate the activation of JNK in rat hippocampus via GluR6 subunit-containing Kainate receptors. ${ }^{16-19}$ To investigate the role of JNK on denitrosylation and activation of procaspase-3 during $\mathrm{I} / \mathrm{R}$, rats were pretreated with SP600125, an inhibitor of JNK. As shown in Figures 3a and b, SP600125 inhibited denitrosylation and activation of procaspase-3. Next, we detected the effect of SP600125 on FasL and Trx-2 level during I/R. The results showed that SP600125 prevented FasL (Figure 3c) and Trx2 (Figure 3d) upregulation induced by $6 \mathrm{~h} \mathrm{I} / \mathrm{R}$. These results further demonstrate that GluR6-JNK pathway is upstream of Fas pathway and Trx-2 system. Inhibition of JNK prevents FasL upregulation and procaspase-3 denitrosylation.
\end{abstract}

FasL mediates denitrosylation and activation of procaspase-3 during I/R. Our previous works have shown that
FasL expression was upregulated after I/R. ${ }^{20,21}$ We hypothesize that the denitrosylation of procaspase- 3 is due to the upregulation of FasL expression. To test this possibility, we first examined the full time courses of FasL level after I/R. The results showed that the level of FasL began to increase (Figure 4a) after $1 \mathrm{~h} \mathrm{I/R}$, which was earlier than that of procaspase-3 denitrosylation. Next, we used the FasL AS-ODNs, which could significantly downregulate the level of FasL in sham rats (Figure 4b). Both the levels of increased FasL and Fas-FasL crosslinking induced by $\mathrm{I} / \mathrm{R}$ were inhibited (Figure 4c). FasL AS-ODNs not only inhibited their expression, but also inhibited the activation and denitrosylation of procaspase-3 (Figures 4d and e). Moreover, FasL AS-ODNs could inhibit the increased expression of Trx-2 (Figure 4f) induced by $6 \mathrm{~h} \mathrm{l} / \mathrm{R}$ in $\mathrm{CA} 1$ region. These results indicate that the increase in FasL expression and the accumulation binding of Fas-FasL lead to the denitrosylation and activation of procaspase-3 during l/R. Trx-2 system may participate in it.

Trx-TrxR system regulates the denitrosylation of procaspase-3 as well as its activation during I/R. Trx-TrxR system can regulate procaspase- 3 denitrosylation. ${ }^{7}$ To verify that it has a role in denitrosylation and activation of procaspase-3 during $\mathrm{I} / \mathrm{R}$, we also first tested the full time courses of Trx-2 level after I/R. The results showed that the level of Trx-2 began to increase after $1 \mathrm{~h}$ I/R (Figure 5a). Auranofin, the TrxR inhibitor, could turn over the denitrosylation and activation induced by $\mathrm{I} / \mathrm{R}$ in hippocumpal CA1 subfields (Figures $5 b$ and $c$ ). To further test the effects of TrxR2 on procaspase-3 denitrosylation and activation, we designed TrxR2 AS-ODNs. The TrxR2 expression was downregulated by $3 \mathrm{~d}$ administration of TrxR2 antisense, $10 \mathrm{nmol} /$ day in sham rats (Figure $5 \mathrm{~d}$ ). It could inhibit the increased expression of TrxR2 induced by $6 \mathrm{~h}$ I/R (Figure 5e). Procaspase-3 denitrosylation ( $3 \mathrm{~h} \mathrm{l} / \mathrm{R})$ and activation $(6 \mathrm{~h} \mathrm{I} / \mathrm{R})$ were also inhibited by TrxR2 antisense (Figures $5 f$ and $g$ ) in hipocampal CA1 subfields.

These results show that once the TrxR is downregulated, Trx cannot exert its function as a denitrosylase to denitrosylate procaspase-3. It also indicates that the Trx-TrxR system mediates denitrosylation and subsequent activation of procaspase-3 during I/R. As Trx2 is only found in mitochondria ${ }^{22}$ and its expression was significantly increased during I/R (Figure 2), mitochondria Trx-TrxR system may have a role in GluR6-KA receptor-coupled procaspase-3 denitrosylation during I/R.

Neuroprotective effects on apoptosis induced by I/R or oxygen and glucose deprivation/reoxygenation (OGD/R) targeting GluR6-FasL-Trx2-procaspase-3 pathway. In order to further demonstrate the important role of GluR6FasL-Trx2 pathway in procaspase-3 activation and cell apoptosis upon I/R, we used a serial of interferences such as lentivirus-short hairprin RNAs (LV-shRNAs), antagonists, or antisenses targeting this pathway. The shRNAs for FasL and TrxR2 were used to knockdown the expression of FasL and TrxR2, respectively, in primary hippocampal neurons (Figure 6). The FasL shRNA and TrxR2 shRNA were successfully infected mediated by lentivirus, which 
a
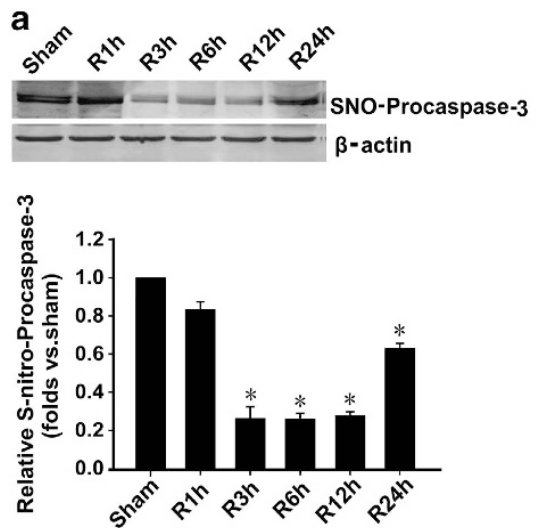
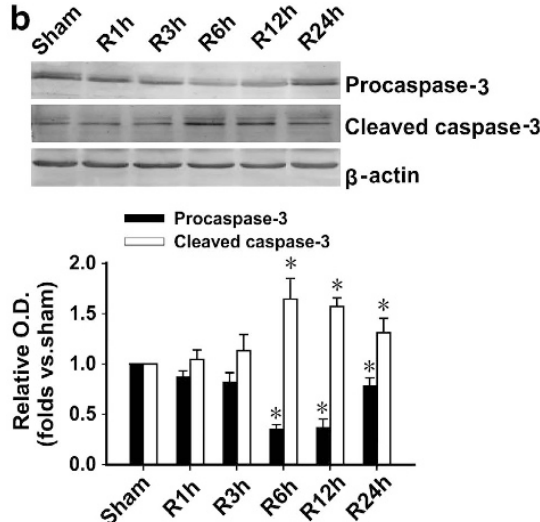

Figure 1 Procaspase-3 is denitrosylated and activated during I/R in hippocampus. (a) Time course analysis of denitrosylation of procaspase-3 levels in hippocampal CA1 derived from sham-treated rats or rats with $15 \mathrm{~min}$ ischemia at various time points $(1,3,6,12$, and $24 \mathrm{~h})$ after reperfusion $(\mathrm{R}) . n=4 ;{ }^{*} P<0.05$ compare with sham group. (b) Time course analysis of procaspase-3 activation (cleaved caspase-3) levels in hippocampal CA1 derived from sham-treated rats or rats with 15 min ischemia at various time points (1, 3 , 6,12 , and $24 \mathrm{~h}$ ) after reperfusion. The samples were processed using the biotin switch method followed by western blotting. Antibodies aginst procaspase-3 and cleaved caspase-3 were used to measure the expression of procaspase-3 and its cleaved large part (17/19 kDa; $n=4 ;{ }^{*} P<0.05$ compare with corresponding sham group). Data are represented as means \pm S.D.

a
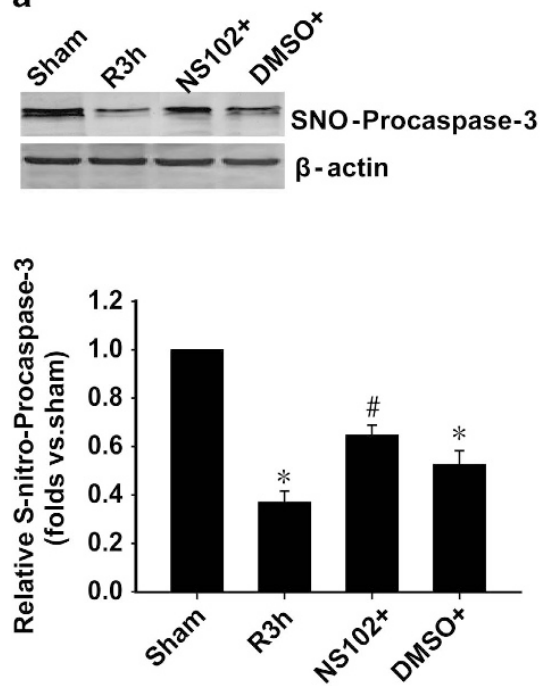

C
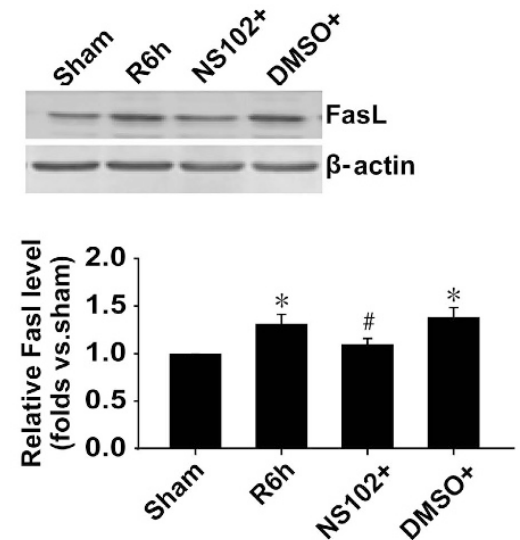

b
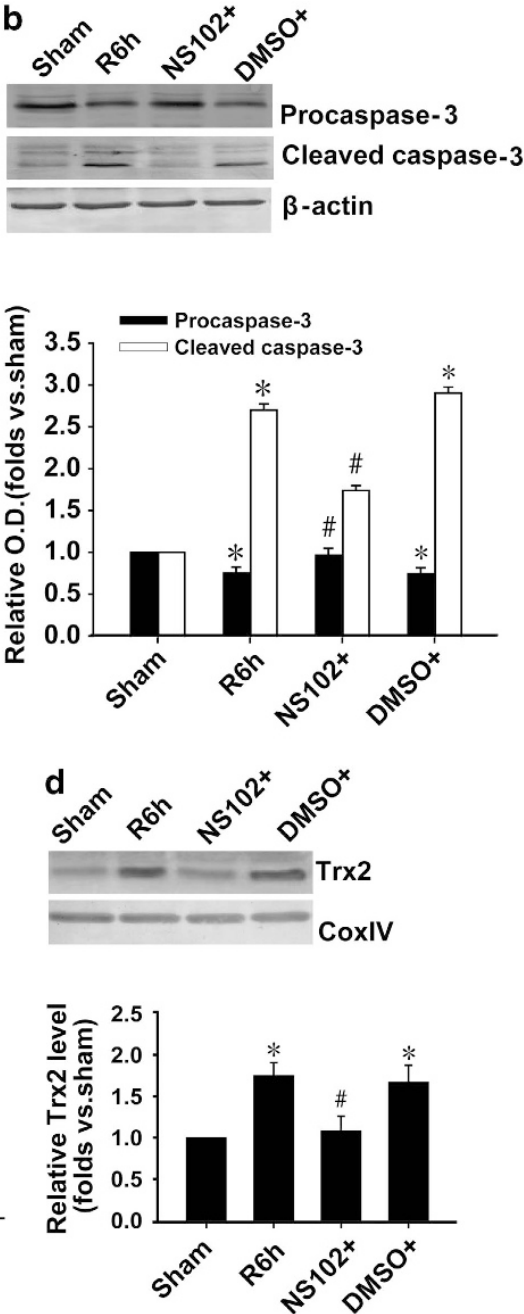

Figure 2 Role of GluR6-containing kainate receptor in the denitrosylation and activation of procaspase-3 during I/R in hippocampus. (a) Procaspase-3 denitrosylation, (b) procaspase-3 activation, (c) expression of FasL, (d) expression of Trx2 were measured followed by $3 \mathrm{~h}$ reperfusion (a) or $6 \mathrm{~h}$ reperfusion (b-d). NS102 (10 mM), an antagonist of GluR6, was administrated 30 min before ischemia. The samples were processed using the biotin switch method followed by western blotting. NS102 almost prevented the denitrosylation and activation of procaspase-3 and the upregulated expression of FasL and Trx-2 after I/R. $n=4$; ${ }^{*} P<0.05$ compare with sham group, ${ }^{\#} P<0.05$ compare with DMSO (I/R) group. Data are represented as means \pm S.D. 
a

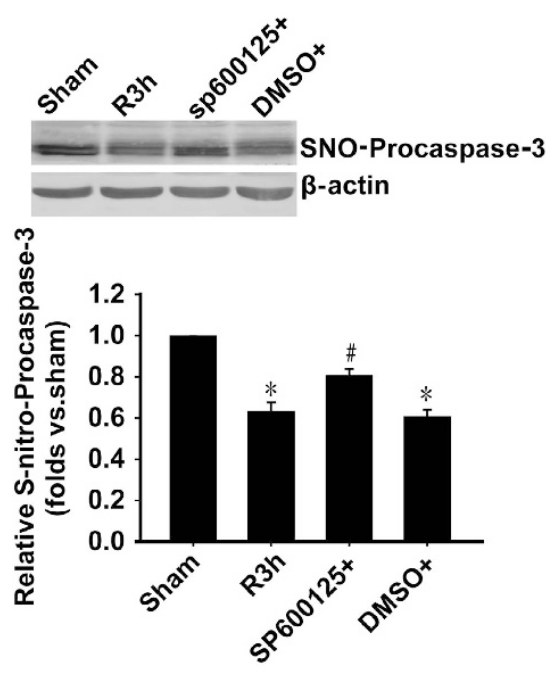

c
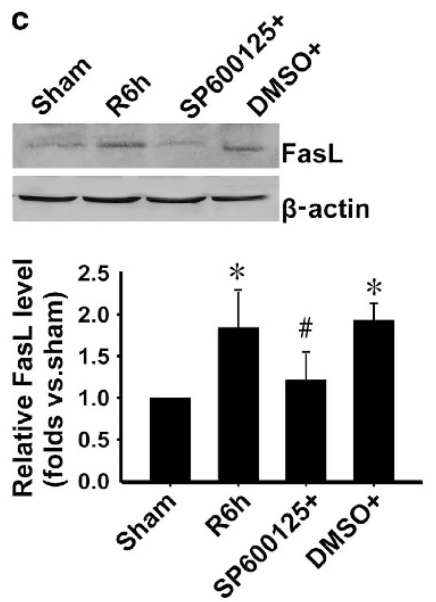

b
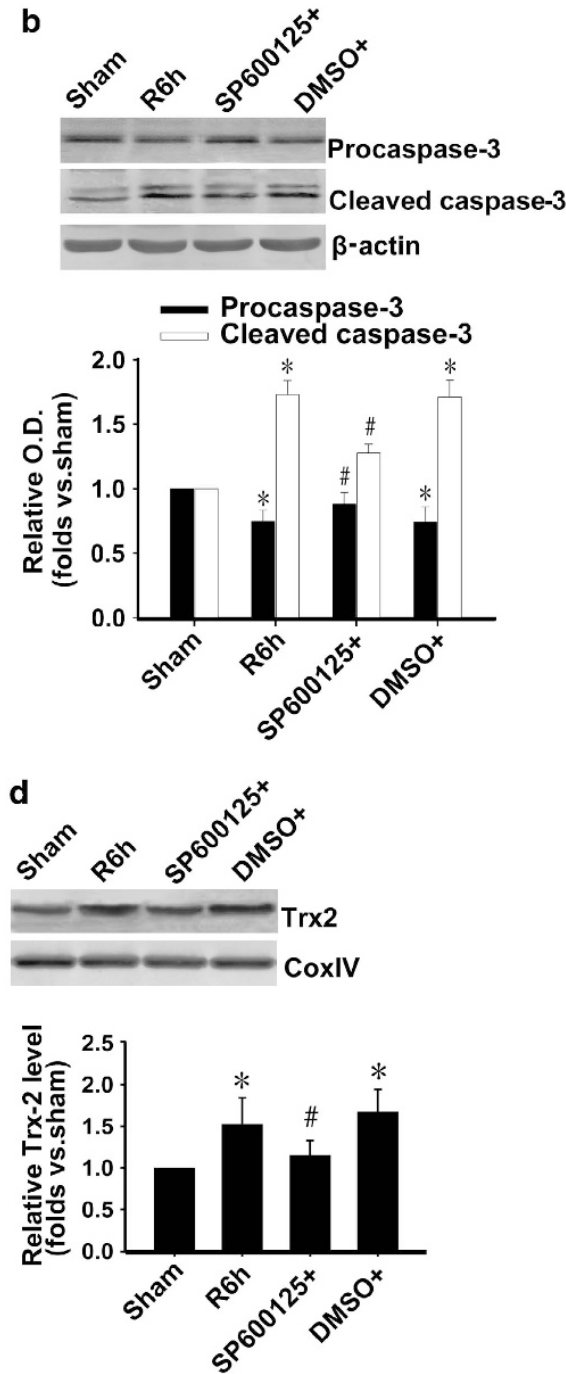

Figure 3 JNK mediates denitrosylation and activation of procaspase-3 during I/R in hippocampus. (a) Procaspase-3 denitrosylation, (b) procaspase-3 activation, (c) level of FasL, (d) level of Trx2 were measured followed by $3 \mathrm{~h}$ reperfusion (a) or $6 \mathrm{~h}$ reperfusion (b-d). SP600125 $(30 \mathrm{mM})$, an inhibitor of JNK, was administrated 20 min before ischemia. The samples were processed using the biotin switch method followed by western blotting. SP600125 inhibited the denitrosylation and activation of procaspase- 3 and the upregulated expression of FasL and Trx-2 after I/R. $n=4 ;{ }^{*} P<0.05$ compare with sham group, ${ }^{\sharp} P<0.05$ compare with DMSO (I/R) group. Data are represented as means \pm S.D.

expresses GFP (Figure 6a) in cultured hippocampal neurons. The levels of protein expression were decreased after $10 \mathrm{~d}$ infection (Figure 6b). LV-FasL shRNA prevented the upregulation of Trx2 level induced by ORG/R6h (Figure 6c). Procaspase-3 denitrosylation (Figure 6d) and activation (Figure 6e) induced by OGD/R were also inhibited by LV-FasL-shRNA and LV-TrxR2-shRNA. DAPI staining results showed that either shRNA protected hippocampal neurons from apoptosis (Figure 6f).

Next, we investigated the roles of antagonists of GluR6 and TrxR, or antisense of FasL and TrxR2 in apoptosis and cell death in rat hippocampal CA1 upon I/R. After $5 \mathrm{~d}, \mathrm{l} / \mathrm{R}$ rats from the sham, I/R, and drug (NS102, FasL AS-ODNs, TrxR2 AS-ODNs, and Auranofin) groups were perfusion-fixed with paraformaldehyde. Cresyl violet staining (Figure 7a) was used to examine the survival of CA1 pyramidal cells in the hippocampus. The numbers of viable cells were counted within a 1-mm length. TUNEL staining was used to examine the apoptosis nuclei with the methyl green (Figure $7 b$ ) after $3 d$ I/R. Sham group or drug-given groups showed round and palely stained nuclei, whereas I/R-induced dead cells showed pyknotic nuclei. The numbers of TUNEL-positive cells were counted within a 1-mm length. These results further indicate that GluR6-FasL-Trx2-Procaspase-3 pathway has an important role in cerebaral I/R-induced cell apoptosis and cell death.

\section{Discussion}

In this study, we demonstrate for the first time that cerebral ischemia-reperfusion increases the activity of procaspase-3, which is associated with decreased S-nitrosylation. One possible molecular mechanisms is that, as shown in Figure 8, cerebral I/R activates GluR6-containing KA receptor, which can increase the expression of FasL and the binding of 

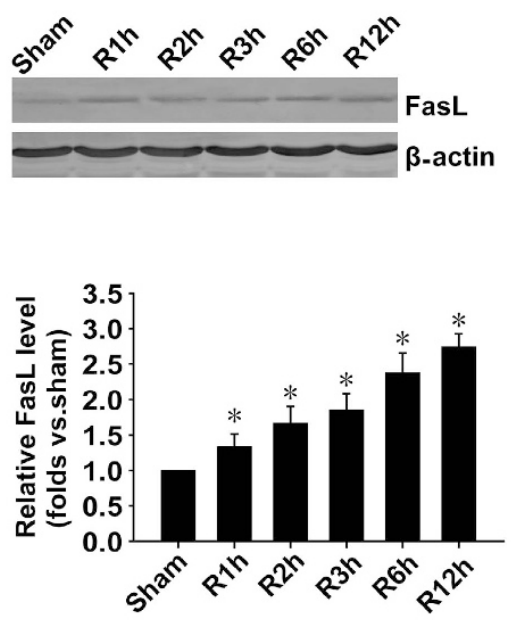

d
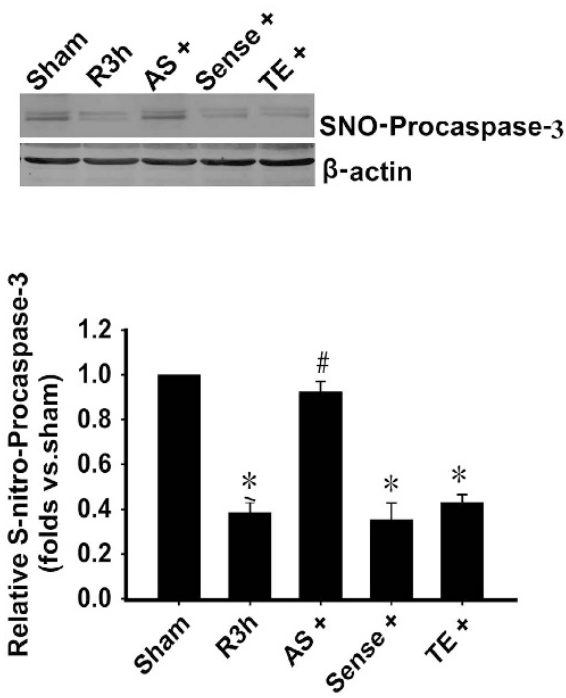

SNO-Procaspase-3 b
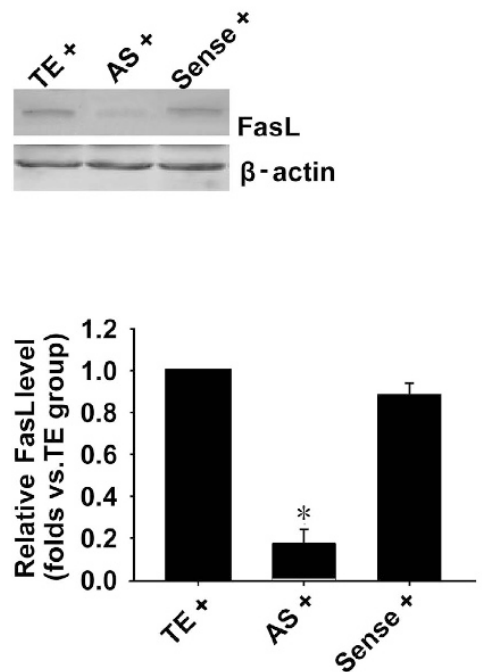

e

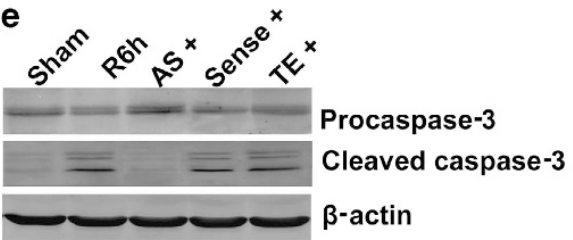

f
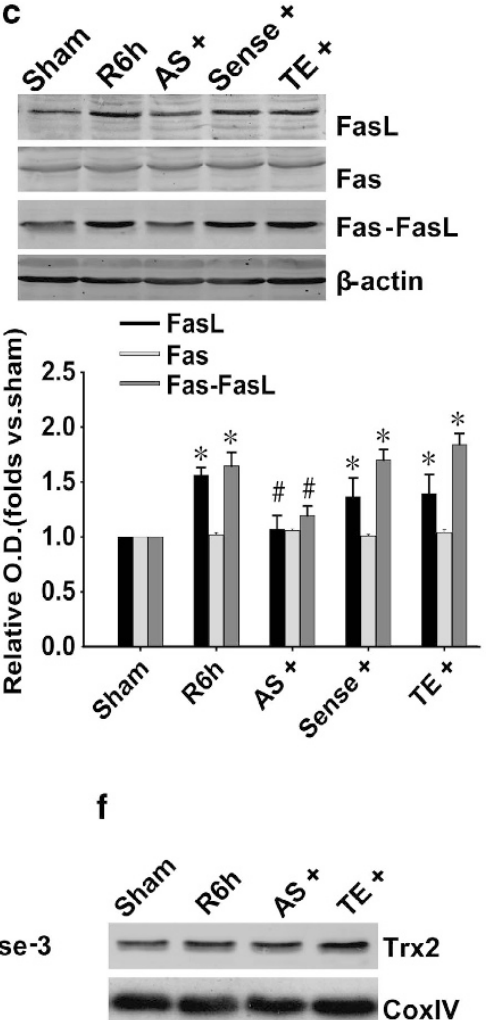

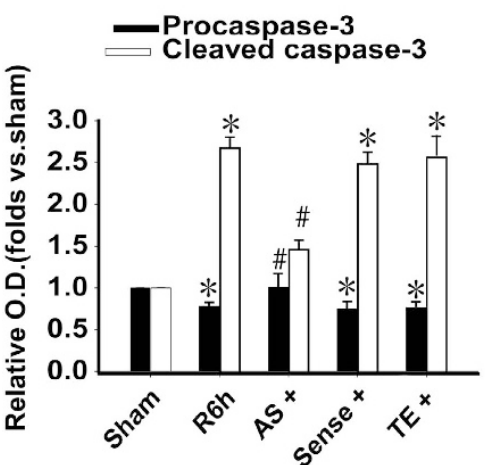

Figure 4 Fas-FasL mediates denitrosylation and activation of procaspase-3 during I/R in hippocampus. (a) Time course analysis of FasL levels in hippocampal CA1 derived from sham-treated rats or rats with 15 min ischemia (I) at various time points $\left(1,2,3,6\right.$, and $12 \mathrm{~h}$ ) after reperfusion (R). $n=4$; ${ }^{\star} P<0.05$ compared with sham group. (b) FasL AS-ODNs (AS) affects FasL expression in sham rats. $10 \mathrm{nmol}$ FasL AS-ODNs, sense-ODNs control (sense), or vehicle (TE) was administrated to the rats every $24 \mathrm{~h}$ for 3 days. FasL-AS significantly decreased FasL expression, whereas sense-ODNs had no effect ( $n=4 ;{ }^{*} P<0.05$ compared with TE group). (c) FasL AS-ODNs (AS) affects FasL expression and Fas-FasL binding during I/R. $10 \mathrm{nmol}$ FasL AS-ODNs, sense-ODNs control (sense), or vehicle (TE) was administrated to the rats every $24 \mathrm{~h}$ for 3 days before ischemia. After $6 \mathrm{~h}$ reperfusion, part of the samples were subjected to coimmunoprecipitation analysis of FasL with Fas followed by western blotting with a FasL antibody, the left samples were immunoblotted with FasL, Fas, $\beta$-actin antibodies, respectively. FasL-AS decreased both FasL expression and Fas-FasL binding, whereas sense-ODNs had no effect $\left(n=4\right.$; ${ }^{*} P<0.05$ compared with sham group, ${ }^{\#} P<0.05$ compared with TE group). (d) FasL AS inhibited denitrosylation of procaspase-3 after $3 \mathrm{~h}$ reperfusion, whereas sense-ODNs had no effect. Samples were processed using the biotin switch method followed by western blotting. $n=4$; ${ }^{*} P<0.05$ compared with sham group, ${ }^{\#} P<0.05$ compared with TE group. (e) FasL AS inhibited procaspase-3 activation after $6 \mathrm{~h}$ reperfusion, whereas sense-ODNs had no effect $\left(n=4\right.$; ${ }^{*} P<0.05$ compared with sham group, ${ }^{\#} P<0.05$ compared with TE group). (f) FasL AS prevented the upregulation of Trx-2 level induced by I/R. $n=4 ;{ }^{*} P<0.05$ compared with sham group, ${ }^{\#} P<0.05$ compared with TE group. Data are represented as means \pm S.D.

FasL with Fas, leading to the activation of Trx-TrxR system. The Trx system mediates procaspase-3 denitrosylation resulting in neuronal apoptosis. Furthermore, our study also indicates that inhibiting the denitrosylation of procaspase-3 exerts neuroprotective effect on I/R.

S-Nitrosylation and denitrosylation, like phosphorylation and dephosphorylation, are a reversibly post-translational protein modification that have important roles in the regulation of protein functions under both physiological and pathological conditions. S-Nitrosylation is thought to exert either pro- or anti-apoptotic effects through regulation of protein function. ${ }^{23}$ For example, the pro-apoptosis proteinase matrix metalloproteinase-2/9 is activated by S-nitrosylation leading to excitotoxic neuronal cell damage. ${ }^{24}$ Our previous studies 
a
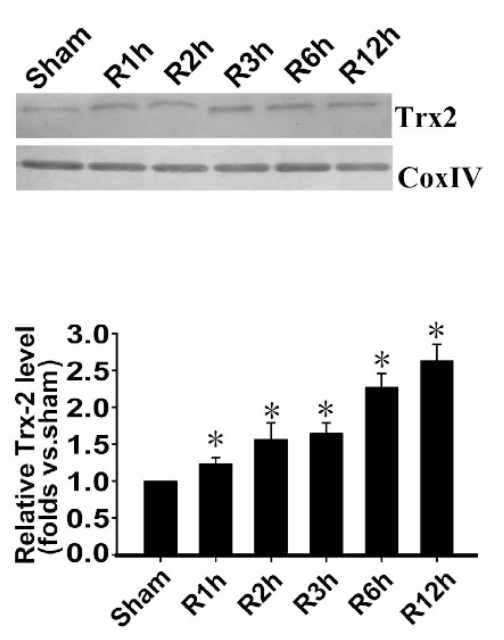

d

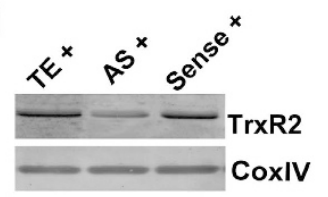

b
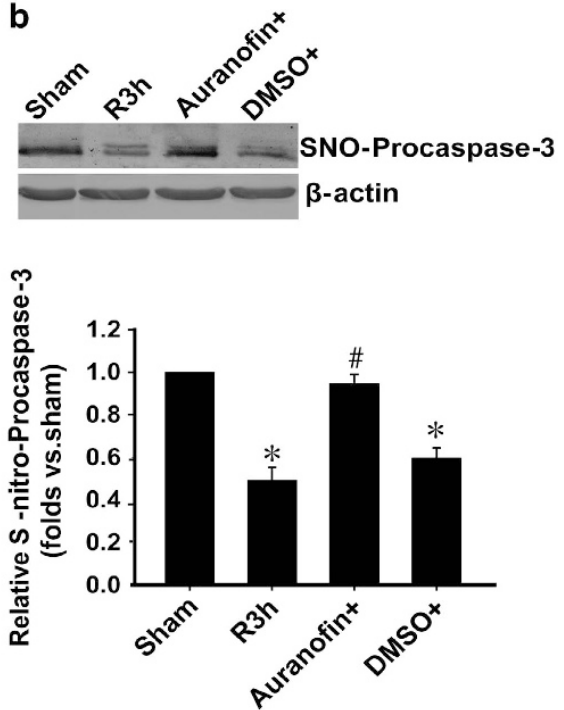

e

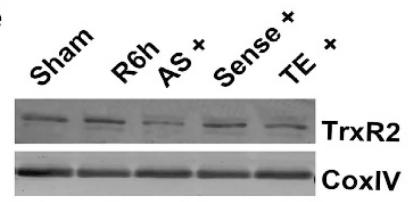

C
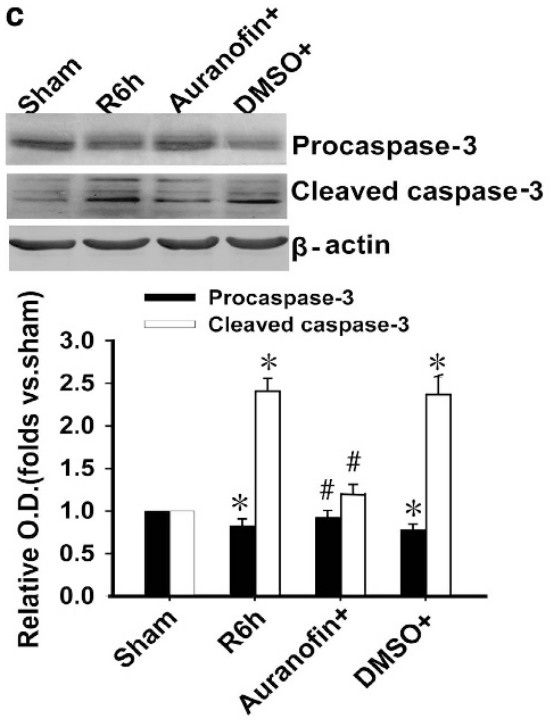

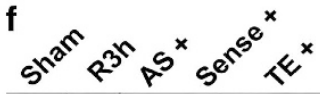

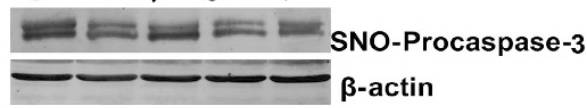

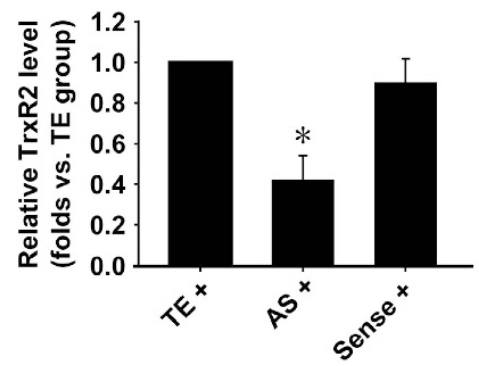

g

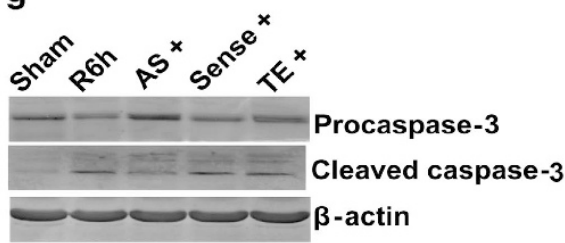

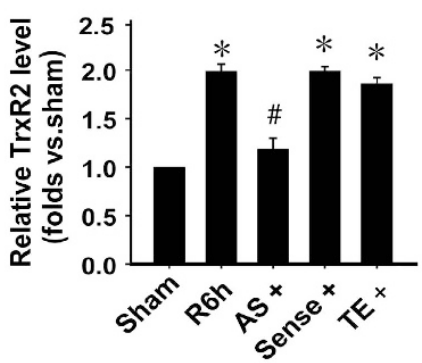
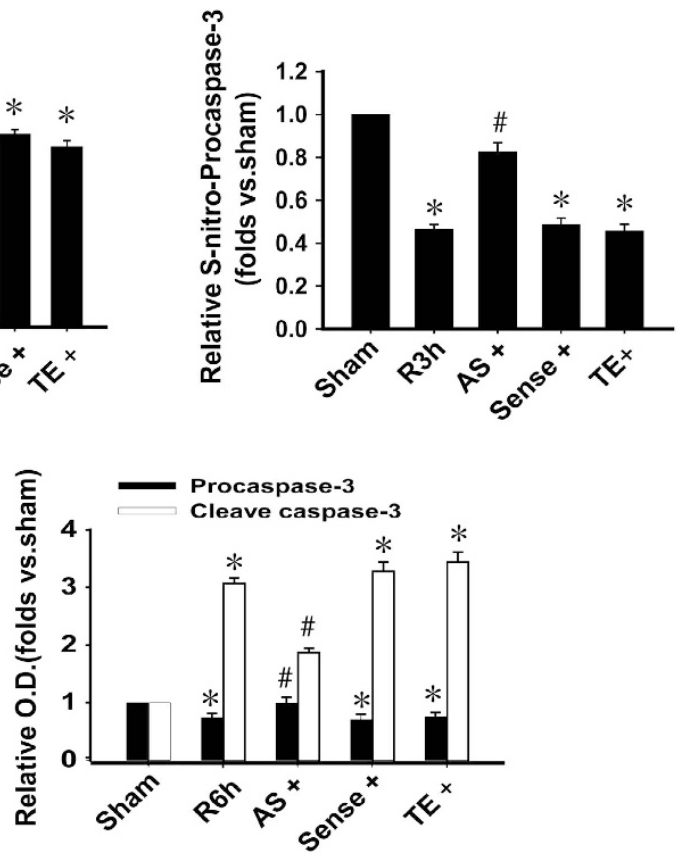

Figure 5 Trx-TrxR system regulates the denitrosylation of procaspase-3 as well as its activation during l/R in hippocampus. (a) Time course analysis of FasL levels in hippocampal CA1 derived from sham-treated rats or rats with 15 min ischemia at various time points $\left(1,2,3,6\right.$, and $12 \mathrm{~h}$ ) after reperfusion (R). $n=4$; ${ }^{*} P<0.05$ compared with sham group. (b) Auranofin protected S-nitrosylation of procaspase-3 after $3 \mathrm{~h}$ reperfusion. Auranofin $(10 \mathrm{mM})$, an inhibitor of TrxR, was administrated 30 min before ischemia. The samples were processed using the biotin switch method followed by western blotting. $n=4$; ${ }^{*} P<0.05$ compared with sham group, ${ }^{\#} P<0.05$ compared with DMSO group. (c) Auranofin inhibited procaspase-3 activation after $6 \mathrm{~h}$ reperfusion ( $n=4 ;{ }^{*} P<0.05$ compared with corresponding sham group, ${ }^{\#} P<0.05$ compared with corresponding DMSO group). (d) TrxR2 AS-ODNs (AS) affects TrxR2 expression in sham rats. $10 \mathrm{nmol}$ TrxR2 AS-ODNs, sense-ODNs control (sense), or vehicle (TE) was administrated to the rats every $24 \mathrm{~h}$ for 3 days. TrxR2 AS significantly decreased TrxR2 expression, whereas sense-ODNs had no effect $\left(n=4 ;{ }^{*} P<0.05\right.$ compared with TE group). (e) TrxR2 AS-ODNs decreased TrxR2 expression during I/R. $10 \mathrm{nmol}$ TrxR2 AS, sense control (sense), or vehicle (TE) was administrated to the rats every $24 \mathrm{~h}$ for 3 days before ischemia. After $6 \mathrm{~h}$ reperfusion, TrxR2 AS decreased TrxR2 expression, whereas sense-ODNs had no effect $\left(n=4\right.$; ${ }^{*} P<0.05$ compared with sham group, ${ }^{\#} P<0.05$ compared with TE group). (f) TrxR2 AS-ODNs protected S-nitrosylation of procaspase-3 after $3 \mathrm{~h}$ reperfusion, whereas sense-ODNs had no effect. The samples were processed using the biotin switch method followed by western blotting. $n=4$; ${ }^{\star} P<0.05$ compared with sham group, ${ }^{\#} P<0.05$ compared with TE group. (g) TrxR2 AS-ODNs inhibited procaspase-3 activation after $6 \mathrm{~h}$ reperfusion, whereas sense-ODNs had no effect $\left(n=4 ;{ }^{*} P<0.05\right.$ compared with sham group, ${ }^{\#} P<0.05$ compared with TE group). Data are represented as means \pm S.D. 
a

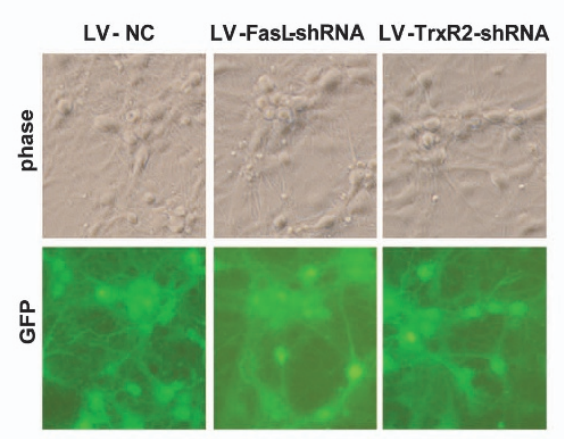

b
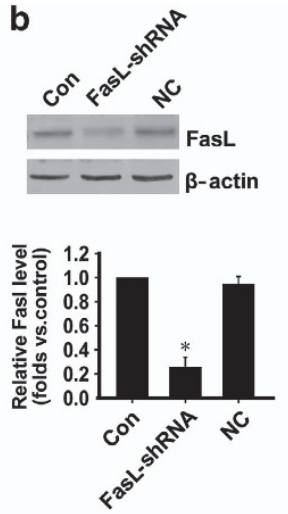
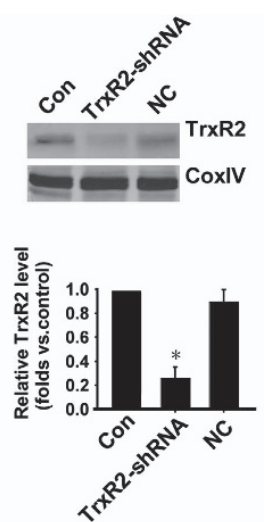

C
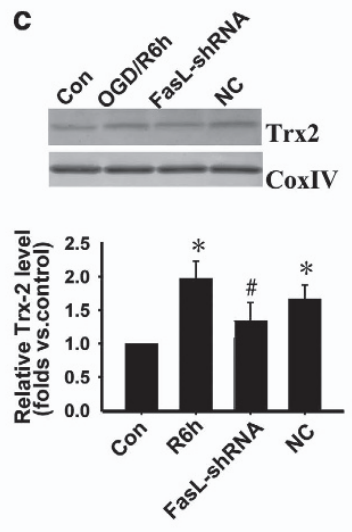

d
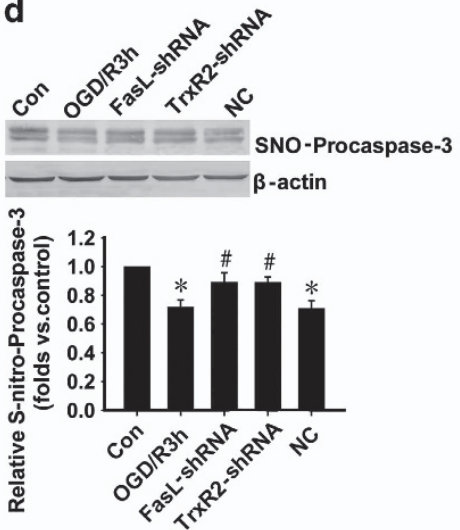

e

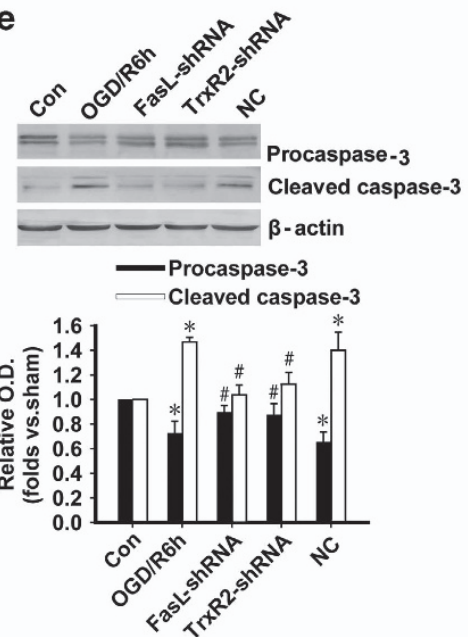

f

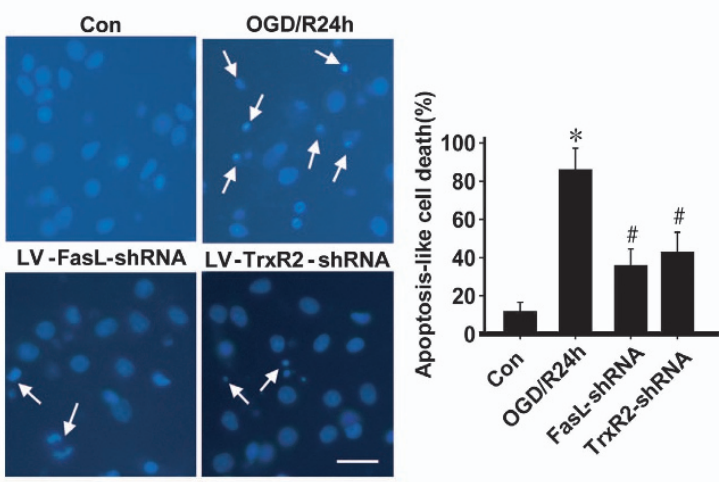

Figure 6 FasL or TrxR2 knockdown inhibits denitrosylation and activation of procaspase-3 after OGD/R in cultured hippocampal neurons. (a) Successful infection of LV-FasL shRNA, LV-TrxR2 shRNA, and LV-NC in primary hippocampal neurons. Scale bar: $20 \mu \mathrm{m}$. (b) The protein expression of FasL and TrxR2 was decreased after 10 days of infection in hippocampal neurons. $n=4$; ${ }^{*} P<0.05$ compared with control group. (c) LV-FasL shRNA prevented the upregulation of Trx2 level induced by OGD/R. $n=4$; ${ }^{*} P<0.05$ compared with sham group, ${ }^{\#} P<0.05$ compared with control group). (d) Procaspase-3 denitrosylation induced by OGD/R ( $3 \mathrm{~h}$ ) was inhibited by LV-FasL shRNA or LV-TrxR2 shRNA. The samples were processed using the biotin switch method followed by western blotting. $n=4$; ${ }^{*} P<0.05$ compared with control group, ${ }^{\#} P<0.05$ compared with NC group. NC, negative control. (e) Procaspase-3 activation induced by OGD/R ( $6 \mathrm{~h}$ ) was inhibited by LV-FasL shRNA and LV-TrxR2 shRNA ( $n=4$; ${ }^{*} P<0.05$ compared with corresponding control group, ${ }^{\#} P<0.05$ compared with corresponding NC group). Data are represented as means \pm S.D. (f) Either LV-FasL or TrxR2 shRNA protected cells from apoptosis. Cells were treated with OGD for $2 \mathrm{~h}$ after 10 days of LV-FasL or TrxR2 shRNA infection, and reperfusion was allowed for $24 \mathrm{~h}$. Typical apoptotic cells with a condensed nucleus were indicated with arrowhead. Apoptic-like cells were expressed as a percentage of the total cells in 10 microscopic fields $(\times 400)$ for DAPI staining. ${ }^{*} P<0.05$ compared with non-infected control group, ${ }^{\#} P<0.05$ compared with OGD/R group). Scale bar: $20 \mu$ m. Data are represented as means \pm S.D.

have demonstrated that $\mathrm{JNK} 3,{ }^{25}$ a pro-apoptosis kinase, is activated by S-nitrosylation during cerebral I/R, which induces neuronal apoptosis. Whereas Akt/PKB, an anti-apoptosis kinase, can be inactivated by S-nitrosylation. ${ }^{25,26}$ In the case of $\mathrm{Bcl}-2$, an anti-apoptosis proteinase, denitrosylation leads to its degradation and reduces its resistance to KA-induced 
a
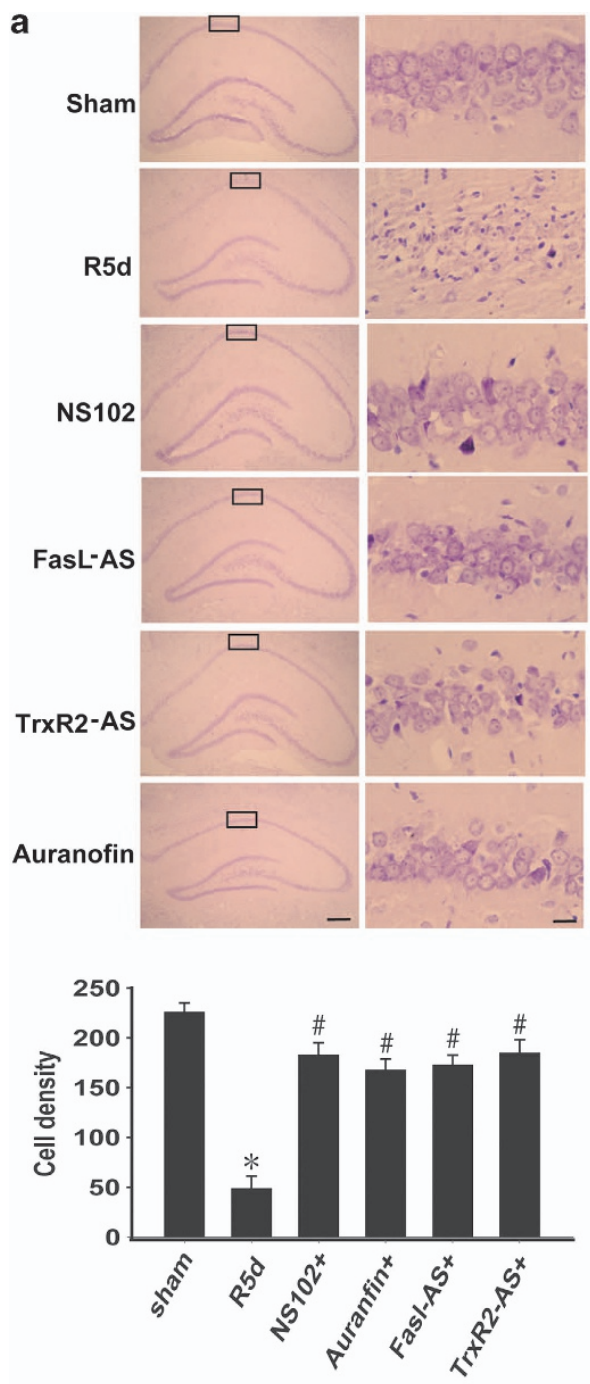

b
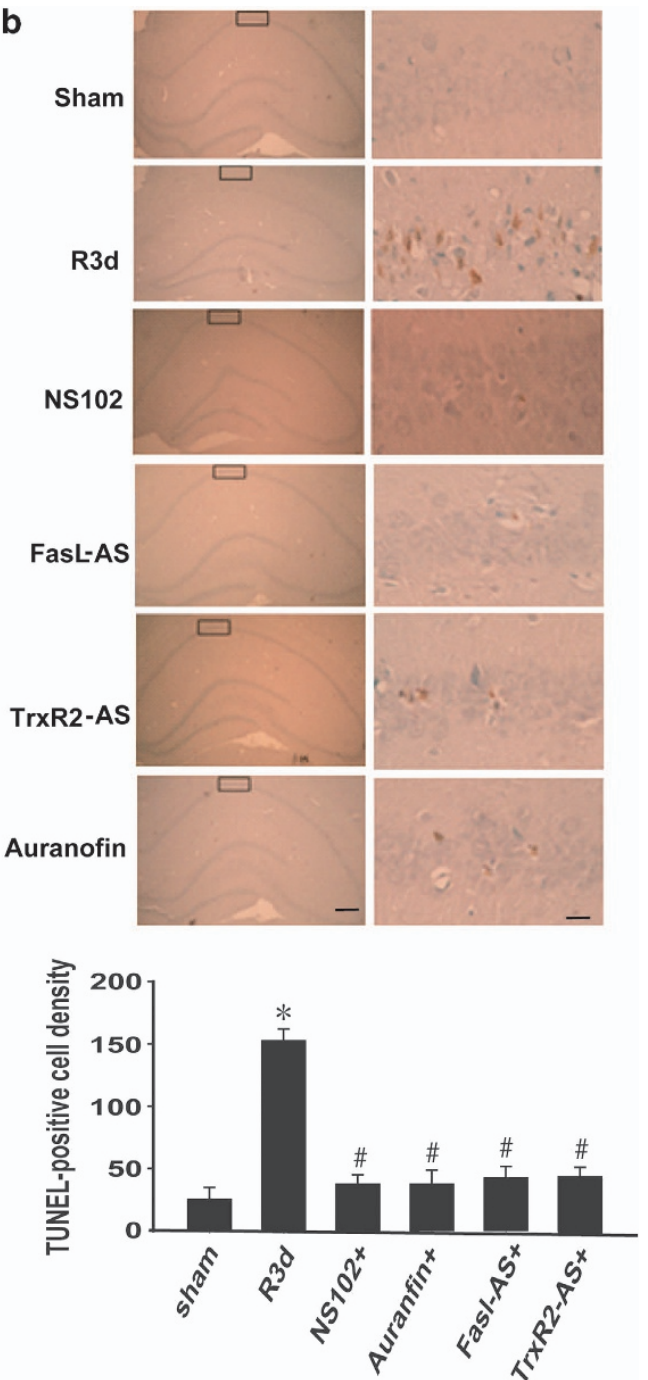

Figure 7 Neuroprotective effects on neuronal injury induced by I/R in hippocampal CA1 region targeting at GluR6-FasL-Trx2-Procaspase-3 pathway. (a) Cresyl violet staining was performed on sections form hippocampus at 5 days reperfusion after $15 \mathrm{~min}$ ischemia. Pretreatment with NS102 (10 mM), Fasl-AS, TrxR2-AS (10 nmol AS was given to the rats every $24 \mathrm{~h}$ for 3 days), or auranofin $(10 \mathrm{mM})$ almost reversed the neuronal death in CA1 area $\left(n=6 ;{ }^{*} P<0.05\right.$ compared with sham group, ${ }^{\#} P<0.05$ compared with I/R $5 \mathrm{~d}$ group). (b) TUNEL staining was performed on sections form hippocampus at 3 days reperfusion after 15 min ischemia. Hippocampal samples counterstained with methyl green were shown. Pretreatment with NS102 (10 mM), Fasl-AS, TrxR2-AS (10 nmol AS was given to the rats every $24 \mathrm{~h}$ for 3 days), or auranofin $(10 \mathrm{mM})$ almost reversed the neuronal apoptosis in CA1 area $\left(n=6\right.$; ${ }^{*} P<0.05$ compared with sham group,${ }^{\#} P<0.05$ compared with $\mathrm{I} / \mathrm{R} 3 \mathrm{~d}$ group). Scale bars, left column, $200 \mu \mathrm{m}$ (magnification $\times 40$ ); right column, $10 \mu \mathrm{m}$ (magnification $\times 400$ ). Data are represented as means \pm S.D.

neuronal apoptosis. ${ }^{7,27,28}$ Here we show that procaspase-3 is also regulated by $\mathrm{S}$-nitrosylation and denitrosylation during cerebral I/R. A certain level of S-nitrosylation is needed to maintain the stability of procaspase- 3 , whereas excess denitrosylation and subsequent cleavage will result in cell apoptosis upon I/R.

Our previous studies have demonstrated a potential signaling pathway in brain injury induced by cerebral I/R. KA receptor subunit GluR6 is activated that facilitates the assembly of GluR6-PSD95-MLK3 signal module, and subsequently activates JNK downstream pathways resulting in neuronal apoptosis. ${ }^{29}$ Activated JNK phosphorylates c-Jun, a nuclear transcription factor, ${ }^{30}$ to increase AP-1 transcriptional activity, which modulates transcription of a number of apoptosis genes such as FasL. We also found that caspase-3 activation is involved in cerebral $1 / R$-induced neuronal apoptosis. Moreover, it has been reported that procaspase-3 is denitrosylated upon activation of the Fas apoptotic pathway. ${ }^{10}$ Results from our current study show that the increased expression of FasL induced by GluR6 and JNK is involved in the denitrosylation and activation of procaspase-3. Decreasing its expression by FasL AS-ODS or shRNA attenuates caspase-3 activation not only in vivo but also in vitro. These results fit well with our hypothesis that KA receptor subunit GluR6-mediated Fas apoptotic pathway results in procaspase-3 denitiosylation and activation-promoting cell apoptosis.

Apoptosis pathway includes intrinsic and extrinsic pathways, also known as death receptor pathway and mitochondrial pathway, respectively. ${ }^{31,32}$ In our study, we show 


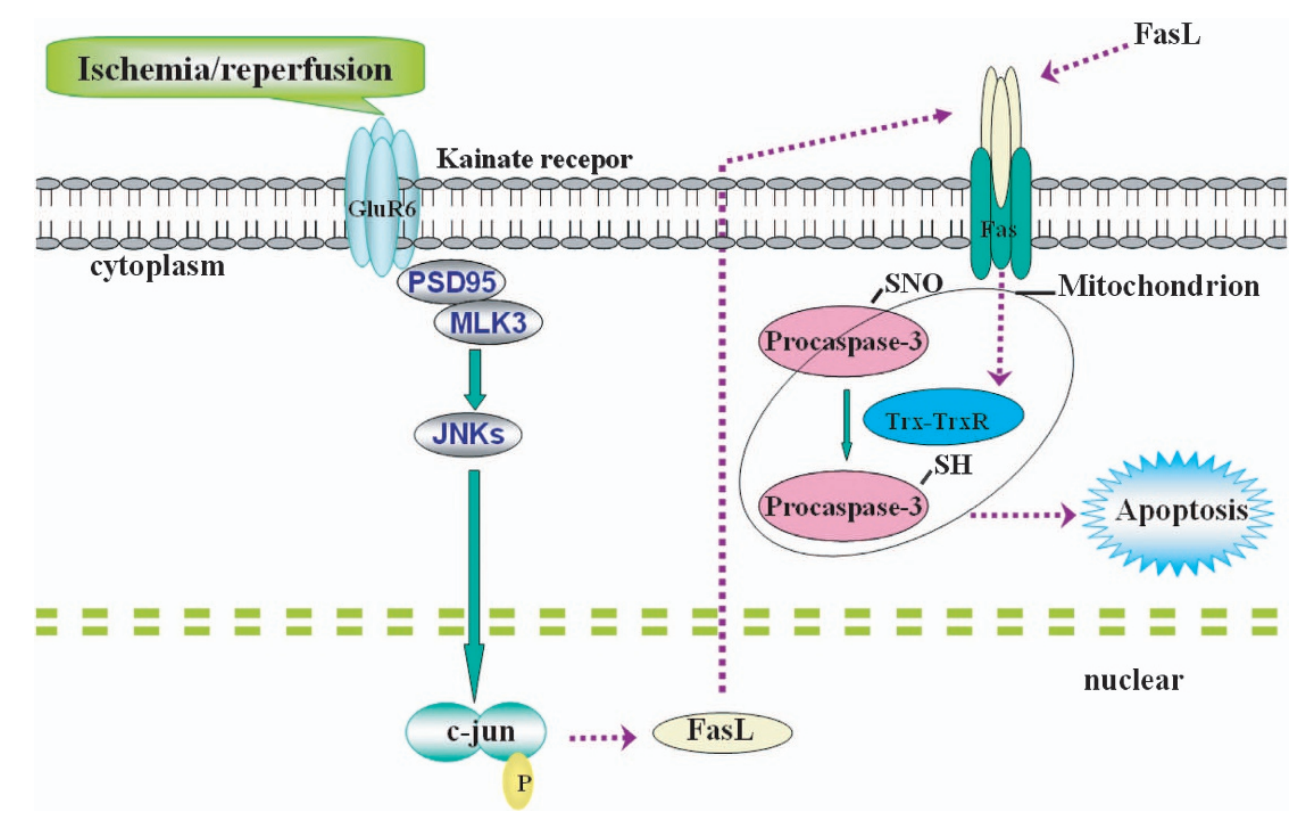

Figure 8 Possible mechanisms for procaspase-3 denitrosylation and activation. Cerebral I/R actives KA receptor subunit GluR6 to induce upregulation of FasL expression, which bind more Fas receptors leading to activation of Trx-TrxR system. Procaspase-3 is then denitrosylated and subsequently activated leading to neuronal apoptosis

that procaspase- 3 is denitrosylated and activated through Fas apoptotic pathway. Fas is a member of the tumor necrosis factor receptor family, which is a classic apoptosis receptor due to its intracellular death domains. ${ }^{33}$ Binding of FasL with Fas induces trimerization, which recruits caspase-8 via the adapter protein Fas-associating protein with death domain (FADD) in the cytoplasm. The FasL-Fas-FADD-procaspase-8 forms death-inducing signaling complex where procaspase-8 autocatalyzes to activate downstream caspase-7 and caspase-3. ${ }^{34}$ Caspase-3 cleaves poly-ADP ribose polymerase to result in cell apoptosis. In addition, procaspase-8 can also promote cell apoptosis via mitochondrial-dependent pathway. ${ }^{35}$

Trx-TrxR system is an important intracellular redox system. Recently, a major enzymatic system of protein denitrosylation is found. ${ }^{7,28}$ Procaspase- 3 can be denitrosylated by Trx2 after Fas stimulation. ${ }^{28}$ Trx proteins have two types, Trx1 and Trx2, which are distributed in different subcellular regions. A substantial amount of Trx1 exists primarily in the cytosol and the nucleus, whereas Trx2 is only found in mitochondria. $^{22}$ Trx1 itself is nitrosylated by S-nitrosoglutathione at Cys73 only after the formation of a Cys32/Cys35 disulfide bond $^{36}$ and Cys73-SNO is specifically transferred a nitrosothiol to the catalytic nucleophile of caspase-3 (Cys163) in vitro to remain its stability. ${ }^{37}$ In vitro Trx-2 S35 directly interacts with procaspase-3 active site and removes NO from procaspase-3 Cys163 SNO. The denitrosylation of target proteins is mediated by catalyzing the conversion of reduced $\operatorname{Trx}-(\mathrm{SH})_{2}$ to oxidized Trx-S $\mathrm{S}_{2}$ Trx-S $\mathrm{S}_{2}$ is reduced by NADPH, which is catalyzed by TrxR. ${ }^{6,7}$ TrxR inhibitors block reduced oxidized thioredoxin to elicit robust S-nitrosylation protein. Genetic or pharmacologic inhibition of either TrxR1 or TrxR2 can make protein S-nitrosylation augmented. Our results are consistent with other results that the expression of Trx2 and
TrxR2 is increased via GluR6 during cerebral I/R. Aunanofin, the inhibitor of both TrxR1 and TrxR2, can inhibit the denitrosylation of procaspase-3 during I/R in vivo. AS-ODNs or shRNA of TrxR2 also specifically attenuate procaspase-3 denitrosylation both in vivo and in vitro. These results agree with our hypothesis that disturbing the Trx-TrxR system might change the whole-cell redox state including many proteins in NO-based signaling pathways. Therefore, it is worthy to study the normal function of Trx-TrxR system in stress or toxic conditions. We also test the time course of Trx-1 expression but it is not changed during I/R (data not shown). So here we focus on the regulation of procaspase- 3 denitrosylation by Trx2-TrxR2 pathway.

Neurodegenerative disorders can be caused by overexpression of either Fas or FasL through uncontrolled apoptosis. The expression and proper functioning of Fas and FasL are critical to cell death and cell survival. In rodent $\mathrm{I} / \mathrm{R}$ model, we have reported that FasL expression is upregulated in line with the time-dependent increase of apoptotic neuron in hippocampus. ${ }^{20,38}$ In the present studies, we show that decreasing the expression of FasL or TrxR2 by AS-ODNs or shRNAs exerts great anti-apoptosis effect by inhibiting the denitrosylation of procaspse-3 in rat hippocampal CA1 subfields.

Taken together, we demonstrate the possible mechanism for procaspase-3 activation: I/R actives KA receptor subunit GluR6 to induce upregulation of FasL expression, which bind more Fas receptors leading to activation of Trx-TrxR system. Procaspase- 3 is then denitrosylated and subsequently activated leading to neuronal apoptosis.

\section{Materials and Methods}

Reagents. Anti-procaspase-3 (\#9662), anti-caspase-3 (\#9661), anti- $\beta$-actin (\#13E5), anti-COXIV (\#4844) bodies were purchased from Cell Signaling 
Technology, Inc. (Cell Signaling, MA, USA). Auranofin (\#sc20476), anti-Fas (\#sc-1023), anti-FasL (\#sc-6237), anti-Trx (\#SC-20146), anti-TrxR1 (\#sC-20147) antibodies were purchased from Santa Cruz Biotechnology, Inc. (Santa Cruz, CA, USA). 6, 7, 8, 9-Tetrahydro-5-nitro-1H-benz [g]indole-2, 3-dione 3-oxime (NS102, \#N179), methyl methylthiomethyl sulfoxide (MMTS, \#177954), (+)-Sodium L-ascorbate (Ascorbate, \#A7631), streptavidin-agarose (\#A1638), Z-Leu-Leu-Glu $\beta$-naphthylamide (Z-Leu-Leu-Glu $\beta N A$, \#C0788) were purchased from SigmaAldrich Co. (St. Louis, MO, USA). (N-(6-(Biotinamido)hexyl)-3'-(2'-pyridyldithio)propionamide (Biotin-HPDP, \#21341) was purchased from Thermo Fisher Scientific Inc. (Rockford, IL, USA). BCIP and NBT were from Promega (Shanghai, China). Anthrapyrazolone (SP600125, \#S1876) was from Beyotime Biontechnology (Haimen, China). Guava TUNEL Kit (\#4500-0121) was purchased from Millipore Co. (Bedford, MA, USA). BCA Kit (\#P0012) and Methyl Green Staining Solution (\#C0115) were purchased from Beyotime Co. (Jiangsu, China). FasL antisenseoligodeoxynucleotides (FasL AS-ODNs), FasL sense oligodeoxynucleotides (FasL S-ODNs), TrxR2 antisenseoligodeoxynucleotides (TrxR2 AS-ODNs), TrxR2 sense oligodeoxynucleotides (TrxR2 S-ODNs), FasL primers, TrxR2 primers, $\beta$-actin primers, COXIV primers were synthesized by Sangon Biotech Co. Ltd. (Shanghai, China). Lentivirus-shRNA (FasL shRNA, TrxR2 shRNA) were designed and synthesized by GenePharma Co. Ltd. (Shanghai, China). All other chemicals were obtained from Sigma unless indicated otherwise.

Animal model of ischemia and reperfusion. Adult male SpragueDawley rats weighed $250 \pm 10 \mathrm{~g}$ were used (Shanghai Experimental Animal Center, Chinese Academy of Science). The experimental procedures were approved by local legislation for ethics of experiments on animals. Transient cerebral ischemia was induced by four-vessel occlusion as described previously. ${ }^{39}$ Briefly, rats were anesthetized with chloral hydrate $(300 \mathrm{mg} / \mathrm{kg}$, intraperitoneally). Then both vertebral arteries were occluded permanently by electrocautery and both carotid arteries were isolated. On the following day, both carotid arteries were occluded with aneurysm clips to induce cerebral ischemia. After $15 \mathrm{~min}$ of the occlusion, the aneurysm clips were removed for reperfusion. Rectal temperature was maintained at $36.5-37.5^{\circ} \mathrm{C}$ during the ischemia and reperfusion process. Rats that lost their righting reflex within $30 \mathrm{~s}$ and pupils dilated and unresponsive to light were selected for the experiments. Sham controls were performed using the same surgical procedures except that the carotid arteries were not occluded.

Drug treatments. NS102 or Auranofin dissolved in dimethlysulfoxide (DMSO) at a concentration of $10 \mathrm{mM}$ was administered $(10 \mu \mathrm{l}$, intracerebroventricular) $30 \mathrm{~min}$ before ischemia. The same dose of DMSO was used as a control. SP600125 (30 $\mu \mathrm{g}$ in $10 \mu \mathrm{l}$ 1\% DMSO) was administrated to the rats $20 \mathrm{~min}$ before ischemia through cerebral ventricular injection. $10 \mathrm{nmol}$ of FasL or TrxR2 antisense oligodeoxynucleotides (AS-ODNs) or sense ODNs (S-ODNs) in $10 \mu \mathrm{l} \mathrm{TE}$ buffer (10 mM Tris- $\mathrm{HCl}, \mathrm{pH}$ 8.0, $1 \mathrm{mM}$ EDTA) were injected to the cerebral ventricle of rats every $24 \mathrm{~h}$ for 3 days. The sequence for FasL AS-ODNs was $5^{\prime}$-CTCTCGGAGTTCTGCCAGCT-3' and for S-ODNs was $5^{\prime}$-AGCTGGCAGAACT CCGAGAG- $3^{\prime}$. The sequence for TrxR2 AS-ODNs was $5^{\prime}$-CAACAACAATCAC CAGGAATG-3' and for S-ODNs was $5^{\prime}$-CACCGATCACCAAGAGATCAA- $3^{\prime}$.

Sample preparation. Rats were decapitated immediately after different time of reperfusion and then the CA1 region of hippocampi were isolated and quickly frozen in liquid nitrogen. Tissues were homogenized in an ice-cold homogenization buffer containing $50 \mathrm{mM}$ MOPS, pH 7.4, $100 \mathrm{mM} \mathrm{KCl}, 320 \mathrm{mM}$ sucrose, $50 \mathrm{mM} \mathrm{NaF}, 0.5 \mathrm{mM} \mathrm{MgCl}, 0.2 \mathrm{mM}$ dithiothreitol, $1 \mathrm{mM}$ EDTA, $1 \mathrm{mM}$ EGTA, $1 \mathrm{mM} \mathrm{Na}_{3} \mathrm{VO}_{4}, 20 \mathrm{mM}$ sodium pyrophosphate, $20 \mathrm{mM} \beta$-phosphoglycerol, $1 \mathrm{mM}$ p-nitrophenyl phosphate, $1 \mathrm{mM}$ benzamidine, $1 \mathrm{mM}$ phenylmethylsulfonyl fluoride, and $5 \mu \mathrm{g} / \mathrm{ml}$ each of leupeptin, aprotinin, and pepstatin A. The homogenates were centrifuged at $800 \times g$ for $10 \mathrm{~min}$ at $4^{\circ} \mathrm{C}$. Supernatants were collected. Protein concentration was determined by the Lowry method. Samples were stored at $-80^{\circ} \mathrm{C}$ and were thawed only once until use.

S-Nitrosylation assay. S-Nitrosylation was detected using the Biotin-Switch method as described by Jaffrey et $a l^{40}$ in which the free thiols in S-nitrosylated proteins were blocked and the S-nitrosothiols were reduced by ascorbic acid sodium salt to yield free thiols, which could be covalently linked to biotin derivatives and assayed using a biotin-based analysis. Briefly, the cells were lysed in HEN buffer (250 mM HEPES, pH 7.7, $1 \mathrm{mM}$ EDTA, $0.1 \mathrm{mM}$ neocuproine, $1 \%$ Nonidet P-40, $150 \mathrm{mM} \mathrm{NaCl}, 1 \mathrm{mM}$ PMSF, protease inhibitor mixture) and the resulting lysates were mixed with an equal volume of MMTS buffer
(25 mM HEPES, pH 7.7, $0.1 \mathrm{mM}$ EDTA, $10 \mu \mathrm{M}$ neocuproine, 5\% SDS, $20 \mathrm{mM}$ MMTS) and incubated at $50^{\circ} \mathrm{C}$ for 20 min with frequent vortexing. After the free MMTS was removed by cold acetone precipitation, the precipitates were resuspended in HENS buffer (25 mM HEPES, pH7.7, $0.1 \mathrm{mM}$ EDTA, $10 \mu \mathrm{M}$ neocuproine, $1 \%$ SDS). After the addition of two volumes of neutralization buffer (20 mM HEPES, pH 7.7, $1 \mathrm{mM}$ EDTA, $100 \mathrm{mM} \mathrm{NaCl}, 0.5 \%$ Triton X-100), the samples were then modified with biotin in the following buffer $(25 \mathrm{mM}$ HEPES, $\mathrm{pH}$ 7.7, $0.1 \mathrm{mM}$ EDTA, $1 \%$ SDS, $10 \mu \mathrm{M}$ neocuproine, $10 \mathrm{mM}$ ascorbic acid sodium salt, and $0.2 \mathrm{mM}$ biotin-HPDP). After free biotin-HPDP was removed by cold acetone precipitation, biotinylated proteins are absorbed to streptavidin-agarose. The streptavidin absorbates were then eluted by $\beta$-mercaptoethanol $(100 \mathrm{mM})$, separated by SDS-polyacrylamide gel electrophoresis (SDS-PAGE) and immunoblotted with an anti-procaspase-3 antibody.

Immunoprecipitation and western blotting analysis. For immunoprecipitation, cytosolic fractions (each containing $400 \mu \mathrm{g}$ of proteins) were diluted fourfold with HEPES buffer containing $50 \mathrm{mM}$ HEPES (pH 7.4), $150 \mathrm{mM} \mathrm{NaCl}$, $10 \%$ glycerol, $1 \%$ Triton X-100, and $1 \mathrm{mM}$ each of EGTA, EDTA, PMSF, and $\mathrm{Na}_{3} \mathrm{VO}_{4}$. The samples were then pre-incubated for $1 \mathrm{~h}$ with $20 \mu \mathrm{l}$ protein A-sepharose CL-4B (Amersham Biosciences, Uppsala, Sweden) and centrifuged to remove any non-specifically adhered proteins from the A-sepharose. The supernatant was then incubated with $2-5 \mu \mathrm{g}$ specific antibodies for $4 \mathrm{~h}$ at $4{ }^{\circ} \mathrm{C}$. After the addition of Protein A-sepharose, the mixture was incubated at $4{ }^{\circ} \mathrm{C}$ for an additional $2 \mathrm{~h}$. Samples were triple washed with HEPES buffer and eluted by SDSPAGE loading buffer then boiled at $100^{\circ} \mathrm{C}$ for $5 \mathrm{~min}$. Western blot analysis was carried out following $15 \%$ SDS-PAGE. Briefly, proteins were electrotransferred onto nitrocellulose membrane (Amersham Biosciences). After blocking for $3 \mathrm{~h}$ in Tris-buffered saline with $0.1 \%$ Tween-20 (TBST) and 3\% bovine serum albumin, membranes were incubated overnight at $4{ }^{\circ} \mathrm{C}$ with primary antibodies in TBST containing $3 \%$ bovine serum albumin. Membranes were then washed and incubated with alkaline phosphatase-conjugated secondary antibodies in TBST for $2 \mathrm{~h}$ and developed using nitro blue tetrazolium/5-bromo-4-chloro-3-indolylphosphate color substrate. The densities of the bands on the membrane were scanned and analyzed with an image analyzer (LabWorks Software; UVP, Upland, CA, USA).

TUNEL staining. TUNEL staining was performed using an ApopTag Peroxidase In Situ Apoptosis Detection Kit (Millipore, Bedford, MA, USA) according to the manufacturer's protocol with minor modifications. Briefly, paraffin-embedded coronal sections were deparaffinized and rehydrated, and then treated with protease $\mathrm{K}$ at $20 \mathrm{mg} / \mathrm{ml}$ for $15 \mathrm{~min}$ at room temperature. The sections were then incubated with reaction buffer containing TdT enzyme and at $37^{\circ} \mathrm{C}$ for $1 \mathrm{~h}$. After washing with stop/wash buffer, the sections were further treated with anti-digoxygenin conjugate for $30 \mathrm{~min}$ at room temperature and the color reaction was subsequently developed in peroxidase substrate. The nuclei were lightly counterstained with $0.5 \%$ methyl green.

Histology. For histological analyses, rats subjected to ischemia post-treatment for 5 days were perfusion-fixed with $4 \%$ paraformaldehyde in $0.1 \mathrm{M}$ phosphate buffer $(\mathrm{pH} 7.4)$ under anesthesia. Paraffin-embedded brain sections $(6 \mu \mathrm{m})$ were then prepared and stained with $0.1 \%(\mathrm{w} / \mathrm{v})$ cresyl violet to assess neuronal damage in the hippocampus. The number of surviving hippocampal CA1 pyramidal cells per $\mathrm{mm}$ was counted as the neuronal density.

Hippocampal neuron cultures and OGD/R. For hippocampal cultures, the hippocampi from E18-19 Sprague-Dawley rats were isolated and dissociated as described previously. ${ }^{41}$ Briefly, cells were plated in culture flask coated with poly-D-lysine at a density of $1-2 \times 10^{6}$ per flask or in 24-well culture plates coated with poly-D-lysine at a density of $1.2-1.6 \times 10^{5}$ cells per well and incubated in Neurobasal medium containing $2 \mathrm{mM}$ GlutaMax and $2 \%$ B27. Thereafter, one-half of the growth medium was replaced twice weekly. Cultures were used after 14 days in vitro (DIV).

For OGD/R, neurons were washed three times with glucose-free Earl's balanced salt solution (EBSS) and incubated in the same EBSS in an anaerobic chamber filled with $5 \% \mathrm{CO}_{2}$ and $95 \% \mathrm{~N}_{2}$ for $2 \mathrm{~h}$. Then neurons were switched back to normal culture conditions for 3 or $6 \mathrm{~h}$ before being harvested. Apoptotic levels after treatments were determined by DAPI. 
Lentiviral-mediated knockdown of FasL and TrxR2 in cultured hippocampal neurons. Hippocampal neurons were infected with lentiviralFasl shRNA (LV-Fasl shRNA, Fasl-shRNA: sense strand: 5'-GUCUAUAUGAG GAACUUUATT- $3^{\prime}$ and antisense strand: $5^{\prime}$-UAAAGUUCCUCAUAUAGACTT-3'), lentivirus-TrxR2 shRNA (LV-TrxR2 shRNA, TrxR2-shiRNA sense strand: $5^{\prime}$-GCAUCACAGUGCUACAUAATT- $3^{\prime}$ and antisense strand: $5^{\prime}$-UUAUGUAGCA CUGUGAUGCTT-3'), or a lentiviral vector that expresses GFP alone (LV-GFP) at DIV 4 for $12 \mathrm{~h}$. OGD experiment was performed at DIV 14

Data analysis and statistics. Values are expressed as mean \pm S.D. Statistical analysis of the results was carried out using the Student's t-test or one-way analysis of the variance (ANOVA) followed by the Duncan's new multiple range method or Newman-Keuls test. $P<0.05$ were considered significant.

\section{Conflict of Interest}

The authors declare no conflict of interest.

Acknowledgements. This work was supported by National Natural Science Foundation of China 30870543 (GYZ), 81273489 (CG), the Natural Science Foundation of Jiangsu Province BK2012582 (CG), Major University Science Research Program of Jiangsu Province 12KJA180008 (CG), a Project Funded by the Priority Academic Program Development of Jiangsu Higher Education Institutions (CG). We thank Professor Jelena Radulovic (Northwestern University) for language proofreading.

1. Stamler JS. Redox signaling: nitrosylation and related target interactions of nitric oxide. Cell 1994; 78: 931-936.

2. Marletta MA. Nitric oxide synthase: aspects concerning structure and catalysis. Cell 1994 78: $927-930$

3. Nathan C, Xie QW. Nitric oxide synthases: roles, tolls, and controls. Cell 1994; 78 915-918.

4. Stamler JS, Simon DI, Osborne JA, Mullins ME, Jaraki O, Michel T et al. S-nitrosylation of proteins with nitric oxide: synthesis and characterization of biologically active compounds. Proc Natl Acad Sci USA 1992; 89: 444-448.

5. Ahern GP, Klyachko VA, Jackson MB. cGMP and S-nitrosylation: two routes for modulation of neuronal excitability by NO. Trends Neurosci 2002; 25: 510-517.

6. Sengupta R, Ryter SW, Zuckerbraun BS, Tzeng E, Billiar TR, Stoyanovsky DA Thioredoxin catalyzes the denitrosation of low-molecular mass and protein S-nitrosothiols. Biochemistry 2007; 46: 8472-8483.

7. Benhar M, Forrester MT, Hess DT, Stamler JS. Regulated protein denitrosylation by cytosolic and mitochondrial thioredoxins. Science 2008; 320: 1050-1054.

8. Gromer S, Arscott LD, Williams CH Jr, Schirmer RH, Becker K. Human placenta thioredoxin reductase. Isolation of the selenoenzyme, steady state kinetics, and inhibition by therapeutic gold compounds. J Biol Chem 1998; 273: 20096-20101.

9. Kumar S. ICE-like proteases in apoptosis. Trends Biochem Sci 1995; 20: 198-202.

10. Mannick JB, Hausladen A, Liu L, Hess DT, Zeng M, Miao QX et al. Fas-induced caspase denitrosylation. Science 1999; 284: 651-654.

11. Nicholson DW, Ali A, Thornberry NA, Vaillancourt JP, Ding CK, Gallant M et al. Identification and inhibition of the ICE/CED-3 protease necessary for mammalian apoptosis. Nature 1995; 376: 37-43.

12. Kim YM, Talanian RV, Billiar TR. Nitric oxide inhibits apoptosis by preventing increases in caspase-3-like activity via two distinct mechanisms. J Biol Chem 1997; 272: 31138-31148.

13. Mohr S, Zech B, Lapetina EG, Brüne B. Inhibition of caspase-3 by S-nitrosation and oxidation caused by nitric oxide. Biochem Biophys Res Commun 1997; 238: 387-391.

14. Tenneti L, D'Emilia DM, Lipton SA. Suppression of neuronal apoptosis by S-nitrosylation of caspases. Neurosci Lett 1997; 236: 139-142.

15. Rössig L, Fichtlscherer B, Breitschopf K, Haendeler J, Zeiher AM, Mülsch A et al. Nitric oxide inhibits caspase-3 by S-nitrosation in vivo. J Biol Chem 1999; 274: 6823-6826.

16. Pei DS, Guan QH, Sun YF, Zhang QX, Xu TL, Zhang GY. Neuroprotective effects of GluR6 antisense oligodeoxynucleotides on transient brain ischemia/reperfusion-induced neuronal death in rat hippocampal CA1 region. J Neurosci Res 2005; 82: 642-649.

17. Pei DS, Wang XT, Liu Y, Sun YF, Guan QH, Wang W et al. Neuroprotection against ischaemic brain injury by a GluR6-9c peptide containing the TAT protein transduction sequence. Brain 2006; 129: 465-479.
18. Gu Z, Jiang Q, Zhang G. c-Jun N-terminal kinase activation in hippocampal CA1 region was involved in ischemic injury. Neuroreport 2001; 12: 897-900.

19. Tian H, Zhang QG, Zhu GX, Pei DS, Guan QH, Zhang GY. Activation of c-Jun $\mathrm{NH} 2$-terminal kinase 3 is mediated by the GluR6.PSD-95.MLK3 signaling module following cerebral ischemia in rat hippocampus. Brain Res 2005; 1061: 57-66.

20. Yu HM, Xu J, Li C, Zhou C, Zhang F, Han D et al. Coupling between neuronal nitric oxide synthase and glutamate receptor 6 -mediated c-Jun $\mathrm{N}$-terminal kinase signaling pathway via S-nitrosylation contributes to ischemia neuronal death. Neuroscience 2008; 155 : 1120-1132.

21. Guan QH, Pei DS, Zhang QG, Hao ZB, Xu TL, Zhang GY. The neuroprotective action of SP600125, a new inhibitor of JNK, on transient brain ischemia/reperfusion-induced neuronal death in rat hippocampal CA1 via nuclear and non-nuclear pathways. Brain Res 2005; 1035: 51-59.

22. Watson WH, Yang X, Choi YE, Jones DP, Kehrer JP. Thioredoxin and its role in toxicology. Toxicol Sci 2004; 78: 3-14.

23. Hess DT, Matsumoto A, Kim SO, Marshall HE, Stamler JS. Protein S-nitrosylation: purview and parameters. Nat Rev Mol Cell Biol 2005; 6: 150-166.

24. Gu Z, Kaul M, Yan B, Kridel SJ, Cui J, Strongin A et al. S-Nitrosylation of matrix metalloproteinases: signaling pathway to neuronal cell death. Science 2002; 297: 1186-1190.

25. Pei DS, Song YJ, Yu HM, Hu WW, Du Y, Zhang GY. Exogenous nitric oxide negatively regulates c-Jun $\mathrm{N}$-terminal kinase activation via inhibiting endogenous NO-induced S-nitrosylation during cerebral ischemia and reperfusion in rat hippocampus. J Neurochem 2008; 106: 1952-1963.

26. Yasukawa T, Tokunaga E, Ota $H$, Sugita $H$, Martyn JA, Kaneki M. S-nitrosylationdependent inactivation of Akt/protein kinase B in insulin resistance. J Biol Chem 2005; 280: $7511-7518$

27. Azad N, Vallyathan V, Wang L, Tantishaiyakul V, Stehlik C, Leonard SS et al. S-nitrosylation of $\mathrm{Bcl}-2$ inhibits its ubiquitin-proteasomal degradation. A novel antiapoptotic mechanism that suppresses apoptosis. J Biol Chem 2006; 281: 34124-34134

28. Benhar M, Forrester MT, Stamler JS. Protein denitrosylation: enzymatic mechanisms and cellular functions. Nat Rev Mol Cell Biol 2009; 10: 721-732.

29. Li C, Xu B, Wang WW, Yu XJ, Zhu J, Yu HM et al. Coactivation of GABA receptors inhibits the JNK3 apoptotic pathway via disassembly of GluR6-PSD-95-MLK3 signaling module in KA-induced seizure. Epilepsia 2010; 51: 391-403.

30. Borsello T, Forloni G. JNK signalling: a possible target to prevent neurodegeneration. Curr Pharm Des 2007; 13: 1875-1886.

31. Falschlehner $\mathrm{C}$, Emmerich $\mathrm{CH}$, Gerlach $\mathrm{B}$, Walczak $\mathrm{H}$. TRAlL signalling: decisions between life and death. Int J Biochem Cell Biol 2007; 39: 1462-1475.

32. Mignotte B, Vayssiere JL. Mitochondria and apoptosis. Eur J Biochem 1998; 252: 1-15.

33. Lambert C, Landau AM, Desbarats J. Fas-beyond death: a regenerative role for Fas in the nervous system. Apoptosis 2003; 8: 551-562.

34. Broughton BR, Reutens DC, Sobey CG. Apoptotic mechanisms after cerebral ischemia. Stroke 2009; 40: e331-e339.

35. Wajant $\mathrm{H}$. The Fas signaling pathway: more than a paradigm. Science 2002; 296: 1635-1636

36. Wu C, Liu T, Chen W, Oka S, Fu C, Jain MR et al. Redox regulatory mechanism of transnitrosylation by thioredoxin. Mol Cell Proteomics 2010; 9: 2262-2275.

37. Mitchell DA, Morton SU, Fernhoff NB, Marletta MA. Thioredoxin is required for S-nitrosation of procaspase-3 and the inhibition of apoptosis in Jurkat cells. Proc Natl Acad Sci USA 2007; 104: 11609-11614.

38. Liu XM, Pei DS, Guan QH, Sun YF, Wang XT, Zhang QX et al. Neuroprotection of Tat-GluR6-9c against neuronal death induced by kainate in rat hippocampus via nuclear and non-nuclear pathways. J Biol Chem 2006; 281: 17432-17445.

39. Pulsinelli WA, Brierley JB. A new model of bilateral hemispheric ischemia in the unanesthetized rat. Stroke 1979; 10: 267-272.

40. Jaffrey SR, Snyder SH. The biotin switch method for the detection of S-nitrosylated proteins. Sci STKE 2001; 2001: pl1.

41. Gao C, Gill MB, Tronson NC, Guedea AL, Guzmán YF, Huh KH et al. Hippocampal NMDA receptor subunits differentially regulate fear memory formation and neuronal signal propagation. Hippocampus 2010; 20: 1072-1082.

Cell Death and Disease is an open-access journal published by Nature Publishing Group. This work is licensed under a Creative Commons Attribution-NonCommercialNoDerivs 3.0 Unported License. To view a copy of this license, visit http://creativecommons.org/licenses/by-nc-nd/3.0/ 